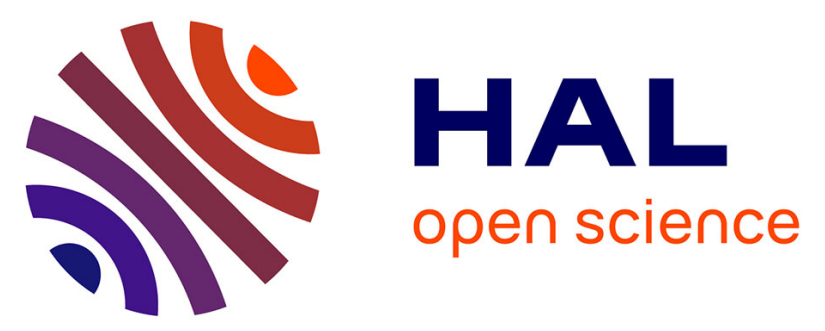

\title{
Electromagnetic properties of concrete: bottom-up modeling from the molecular scale
}

\author{
Tulio Honorio, Farid Benboudjema, Thierry Bore, Helena Carasek, Oswaldo \\ Cascudo, Mehdi Ferhat, Eric Vourc'H
}

\section{- To cite this version:}

Tulio Honorio, Farid Benboudjema, Thierry Bore, Helena Carasek, Oswaldo Cascudo, et al.. Electromagnetic properties of concrete: bottom-up modeling from the molecular scale. RILEM-SC2020 Ambitioning a Sustainable Future for Built Environment: Comprehensive Strategies for Unprecedented Challenges, Mar 2020, Guimarães, Portugal. hal-02500081

\section{HAL Id: hal-02500081 \\ https://hal.science/hal-02500081}

Submitted on 5 Mar 2020

HAL is a multi-disciplinary open access archive for the deposit and dissemination of scientific research documents, whether they are published or not. The documents may come from teaching and research institutions in France or abroad, or from public or private research centers.
L'archive ouverte pluridisciplinaire HAL, est destinée au dépôt et à la diffusion de documents scientifiques de niveau recherche, publiés ou non, émanant des établissements d'enseignement et de recherche français ou étrangers, des laboratoires publics ou privés. 


\title{
Electromagnetic properties of concrete: bottom-up modeling from the molecular scale
}

\author{
Tulio Honorio ${ }^{1}$, Farid Benboudjema ${ }^{1}$, Thierry Bore ${ }^{2}$, Helena Carasek ${ }^{3}$, \\ Oswaldo Cascudo ${ }^{3}$, Mehdi Ferhat ${ }^{4}$, and Eric Vourc' ${ }^{4}$ \\ 1 Université Paris-Saclay, ENS Paris-Saclay, CNRS, LMT - Laboratoire de \\ Mécanique et Technologie, 94235, Cachan, France \\ tulio.honorio-de-faria@ens-paris-saclay.fr \\ 2 School of Civil Engineering, The University of Queensland, Brisbane, Australia \\ 3 Universidade Federal de Goiás, Escola de Engenharia Civil e Ambiental, \\ PPG-GECON, Goiânia, Brazil \\ 4 SATIE, UMR CNRS 8029, ENS Paris Saclay, Université Paris Saclay, Cachan, \\ France
}

\begin{abstract}
The electromagnetic response of concrete can be used to the non-destructive testing of structures and to follow early-age property development. Fundamental understanding of the physical origins of the electromagnetic response of cement-based materials is critical to reduce the empirism in the interpretation of electromagnetic-based techniques. The pore solution is the main contribution to the electrical conductivity and dielectric response of porous geomaterials. Specific ion effects are known to impact the dynamics of ions in aqueous salt solutions. In this context, molecular dynamics (MD) simulation is a well-suited technique to compute and understand how the ionic composition of the pore solution affects the electromagnetic properties of concrete. Here, we discuss recent results of MD simulation on bulk solution mimicking concrete pore solution. Then, we upscale the information from the molecular scale up to the concrete scale in order to provide a multiscale model of the electrical conductivity and frequency-dependent dielectric response of cement-based materials.
\end{abstract}

Keywords: Dielectric properties - Electrical conductivity - Frequencydependent response $\cdot$ Molecular dynamics simulation $\cdot$ Micromechanics.

\section{Introduction}

Measurements of the electromagnetic response of concrete have been extensively used for monitoring purposes. The related techniques, including resistivity and high-frequency electromagnetic (ground-penetrating radar, L-band remote sensing, capacitive probe, etc) measurements, are non-destructive. Electromagnetic probing of concrete can also be used to follow the development of the material property at early age. Fundamental understanding of the electromagnetic properties of the phases present in cement-based materials, especially the pore 
solution, is critical to enhance the performance of these techniques and to improve the confidence in the corresponding results.

In this paper, we propose a multiscale strategy to model both resistivity and dielectric response of concrete informed by the dynamics of water and ion at the molecular scale [9]. We have previously deployed molecular simulation to get information with atomic-level detail on the physical origins of various properties of microporous materials $[12,13,4]$ The dynamics and dielectric response based on molecular simulations of bulk system mimicking pore solutions are discussed. The frequency-dependent response of the susceptibility and conductivity of the pore solutions are analyzed. Micromechanics theory is then deployed to upscale the information obtained from molecular dynamics simulations.

\section{Theory and Calculation}

\subsection{Electrical conductivity and Dielectric response from fundamentals}

For a system with $n$ particles $i$ with charge $q_{i}$, the total system polarization $\boldsymbol{P}$ is the sum of the polarization (or dipole moment) $\boldsymbol{\mu}_{\boldsymbol{i}}(t)$ of the each particle $i$ at time $t$ [16]: $\boldsymbol{P}(t)=\sum_{i=1}^{n} \boldsymbol{\mu}_{\boldsymbol{i}}(t)=\sum_{i=1}^{n} q_{i} \boldsymbol{r}_{\boldsymbol{i}}(t)$, with $\boldsymbol{r}_{\boldsymbol{i}}(t)$ being the position of the (center of) particle $i$. The total system polarization $\boldsymbol{P}$ is related to the electric field $\boldsymbol{E}$ via the complex frequency-dependent dielectric susceptibility $\chi(f)=$ $\chi^{\prime}(f)-i \chi^{\prime \prime}(f)$ by $\boldsymbol{P}(f)=\chi(f) \epsilon_{0} \boldsymbol{E}(f)$, where $\epsilon_{0}$ is the vacuum permittivity. The dielectric susceptibility $\chi(f)$ can be computed from the auto-correlation of equilibrium polarization fluctuations using [14]:

$$
\chi(f)=-\frac{1}{3 V k_{B} T \epsilon_{0}} \int_{0}^{\infty} e^{-2 \pi i f t}\langle\boldsymbol{P}(0) . \dot{\boldsymbol{P}}(t)\rangle d t
$$

where $V$ and $T$ are the volume and temperature of the system, respectively; $k_{B}$ is the Boltzmann constant and $\dot{\boldsymbol{P}}(t)$ is the time-derivative of the total polarization.

For a salt aqueous solution, the total polarization is the sum of the contributions of the water $\boldsymbol{P}_{W}$ and ionic $\boldsymbol{P}_{I}$ polarizations: $\boldsymbol{P}=\boldsymbol{P}_{W}+\boldsymbol{P}_{I}$. The ionic current $\boldsymbol{J}_{I}$ is related to the ionic polarization by: $\boldsymbol{J}_{I}(t)=\dot{\boldsymbol{P}}_{I}(t)$. This ionic current $\boldsymbol{J}_{I}$ is linked to the electric field $\boldsymbol{E}$ via the frequency-dependent ionic conductivity $\sigma(f)=\sigma^{\prime}(f)-i \sigma^{\prime \prime}(f)$ by

$$
\boldsymbol{J}_{I}(f)=\sigma(f) \epsilon_{0} \boldsymbol{E}(f)
$$

The frequency-dependent ionic conductivity can be computed using the cross correlations $[2,17]$ :

$$
\sigma(f)=\frac{1}{3 V k_{B} T} \int_{0}^{\infty} e^{-2 \pi i f t}\left\langle\boldsymbol{J}_{I}(0) . \dot{\boldsymbol{P}}(t)\right\rangle d t
$$

The contributions of water-water, ion-water and ion-ion correlations can be separated using the following auto- and cross-correlations functions of the water polarization and ionic current can be defined [17]: 


$$
\begin{gathered}
\phi_{W}(t)=\frac{\left\langle\boldsymbol{P}_{W}(0) \cdot \boldsymbol{P}_{W}(t)\right\rangle}{3 V k_{B} T \epsilon_{0}} \\
\phi_{I W}(t)=\frac{\left\langle\boldsymbol{P}_{W}(0) \cdot \boldsymbol{J}_{I}(t)-\boldsymbol{J}_{I}(0) \cdot \boldsymbol{P}_{W}(t)\right\rangle}{3 V k_{B} T \epsilon_{0}} \\
\phi_{I}(t)=\frac{\left\langle\boldsymbol{J}_{I}(0) \cdot \boldsymbol{J}_{I}(t)\right\rangle}{3 V k_{B} T \epsilon_{0}}
\end{gathered}
$$

The regularized susceptibility $\Delta \chi(f)$ can be decomposed into three separate contributions:

$$
\Delta \chi(f)=\chi_{W}(f)+\chi_{I W}(f)+\Delta \chi_{I}(f)
$$

where

$$
\begin{gathered}
\chi_{W}(f)=\phi_{W}(0)-i 2 \pi f \int_{0}^{\infty} e^{-2 \pi i f t} \phi_{W}(t) d t \\
\chi_{I W}(f)=-2 \int_{0}^{\infty} e^{-2 \pi i f t} \phi_{I W}(t) d t \\
\Delta \chi_{I}(f)=-\frac{i}{2 \pi f} \int_{0}^{\infty}\left(e^{-2 \pi i f t}-1\right) \phi_{I}(t) d t
\end{gathered}
$$

The frequency-dependent ionic conductivity can be decomposed into the two terms:

$$
\sigma(f)=\sigma_{I W}(f)+\sigma_{I}(f)
$$

with

$$
\begin{gathered}
\sigma_{I W}(f)=-i 2 \pi f \epsilon_{0} \int_{0}^{\infty} e^{-2 \pi i f t} \phi_{I W}(t) d t \\
\sigma_{I}(f)=\epsilon_{0} \int_{0}^{\infty} e^{-2 \pi i f t} \phi_{I}(t) d t
\end{gathered}
$$

Therefore, the static conductivity can be computed via $\sigma(f=0)=\sigma_{I}(f=0)=$ $\epsilon_{0} \int_{0}^{\infty} \phi_{I}(t) d t$.

\subsection{Homogenization of the electrical conductivity and dielectric permittivity}

Micromechanics is the study of the mechanical, thermal, electromagnetic and mass transport behavior of the materials with a microstructure [15]. The homogenization of the electrical conductivity is analogous to the homogenization of the thermal conductivity, dielectric permittivity, and diffusion coefficient [18]. In this section, we recall three homogenization schemes - namely Mori-Tanaka 
(MT, or Maxwell-Garnett) and Self-Consistent (SC, or Bruggeman) schemes that we have extensively used (e.g. $[20,6,5,8]$ ) to estimate the effective properties of cement-based materials accounting for the hierarchical microstructure of the material. With these homogenization schemes, it is possible to account for the random nature of cement-based materials microstructure and interactions among the different phases in a simplified way. The estimations are not computer-intensive compared to numerical homogenization based on finite element methods.

In a matrix/inclusion morphology, for a $(N+1)$-phase heterogeneous material with $N$ isotropic spherical inclusions randomly distributed in a representative elementary volume, the Mori-Tanaka estimation of the effective electrical conductivity $\sigma^{M T}$ (resp. dielectric permittivity) can be computed from [18]:

$$
\frac{\sigma^{M T}-\sigma_{0}}{\sigma^{M T}+2 \sigma_{0}}=\sum_{r=1}^{N} f_{r} \frac{\sigma_{r}-\sigma_{0}}{\sigma_{r}+2 \sigma_{0}}
$$

where $f_{r}$ is the volume fraction of the phase $r$, and the subscript ${ }_{0}$ denotes the (isotropic) matrix phase.

In a polycrystalline-like morphology, for a $N$-phase heterogeneous materials with $N$ isotropic equiaxed inclusions randomly distributed in representative elementary volume, the Self-Consistent estimation of the effective electrical conductivity $\sigma^{S C}$ (resp. dielectric permittivity) can be computed from the implicit formula [18]:

$$
\sum_{r=1}^{N} f_{r} \frac{\sigma_{r}-\sigma^{S C}}{\sigma_{r}+2 \sigma^{S C}}=0
$$

The presence of interphases (i.e. the volume in-between two phases - in contrast with an "interface", which is a surface between two surfaces) can be modeled with the Generalized Self-Consistent (GSC) scheme based on the compositesphere morphology. For the sake of simplicity, ITZ is not taken into account here. See [11] for a detailed analysis of the influence of ITZ in the electrical conductivity of mortars.

\section{Results}

\subsection{Dielectric response and conductivity from molecular dynamics}

Figure 1 shows the age-dependent ionic composition of the pore solution based on the experimental results of Vollpracht et al. [19] for CEM I system with $w / c=0.4$. Bulk solution with the selected compositions is modeled using classical molecular dynamics as detailed in Honorio et al. [9]. PC1 and PC2 correspond to typical pore solution at the very early age (before setting). PC3 and PC4 correspond to pore solution at early-age per se (after setting), and PC5-PC7 correspond to late ages pore solutions. 


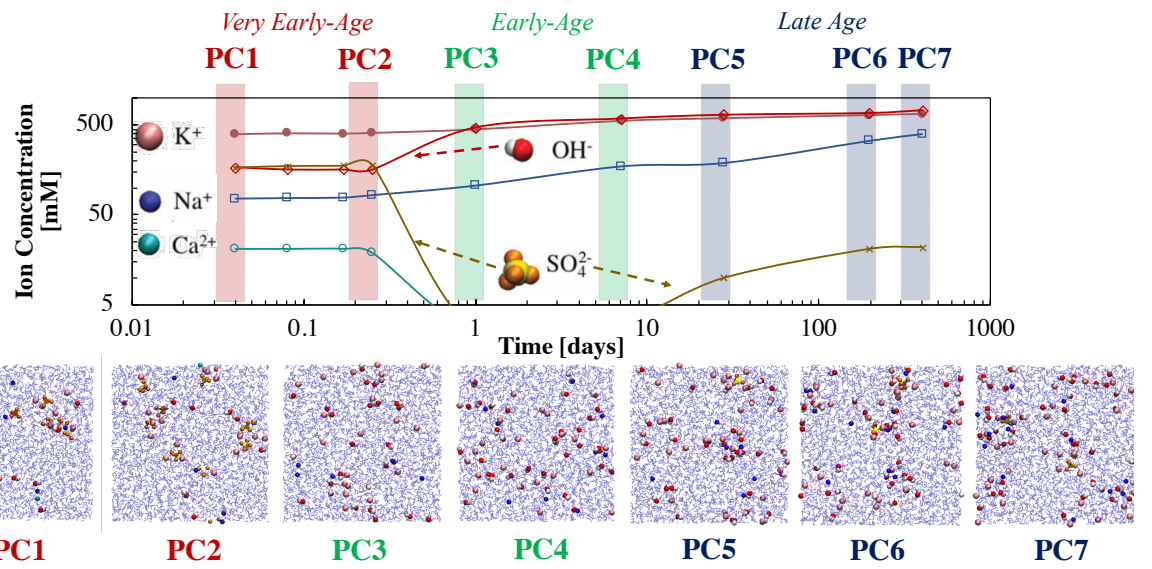

Fig. 1. Age-dependent ionic composition of the pore solution based on the experimental results of Vollpracht et al. [19]. Snapshot of the atomic configuration obtained in the MD simulations reported in ref. [9]

The frequency-dependent DC-conductivity and dielectric spectra of the agedependent pore solutions are shown in Fig. 2. As discussed in ref. [10], the contribution of water-water interactions, are predominant in the dielectric response. The dielectric spectra can be fitted using the Cole-Cole model with the parameters gathered in Table 1. For comparison, we also provide the fits for SPC/E water that are in agreement with experiments [1] and previous simulation with $\mathrm{SPC} / \mathrm{E}$ water model [17]. We observe that the variability of the ion composition of the pore solution leads to significant variations of the dielectric response: on the order of tens units of relative dielectric permittivity.

Table 1. Cole-Cole fit parameters from MD simulations of pore solution and SPC/E water [10]. The variability/uncertainty associated with the fittings were computed suing Pearson's correlations.

\begin{tabular}{|c|c|c|c|}
\hline & $\epsilon_{C C}[-]$ & $\tau_{C C}[\mathrm{ps}]$ & $\alpha[-]$ \\
\hline \hline PC1 & $73.6 \pm 2.9$ & $9.97 \pm 0.97$ & $0.000 \pm 0.060$ \\
PC2 & $67.1 \pm 2.6$ & $10.26 \pm 0.97$ & $0.009 \pm 0.060$ \\
PC3 & $73.9 \pm 3.2$ & $12.55 \pm 1.33$ & $0.000 \pm 0.061$ \\
PC4 & $60.7 \pm 2.7$ & $9.33 \pm 1.14$ & $0.000 \pm 0.050$ \\
PC5 & $56.0 \pm 2.1$ & $9.80 \pm 0.96$ & $0.040 \pm 0.056$ \\
PC6 & $55.2 \pm 2.4$ & $9.33 \pm 1.27$ & $0.098 \pm 0.055$ \\
PC7 & $60.4 \pm 3.0$ & $10.30 \pm 1.52$ & $0.052 \pm 0.063$ \\
\hline SPC/E & $68.2 \pm 2.7$ & $8.35 \pm 0.94$ & $0.033 \pm 0.055$ \\
\hline
\end{tabular}



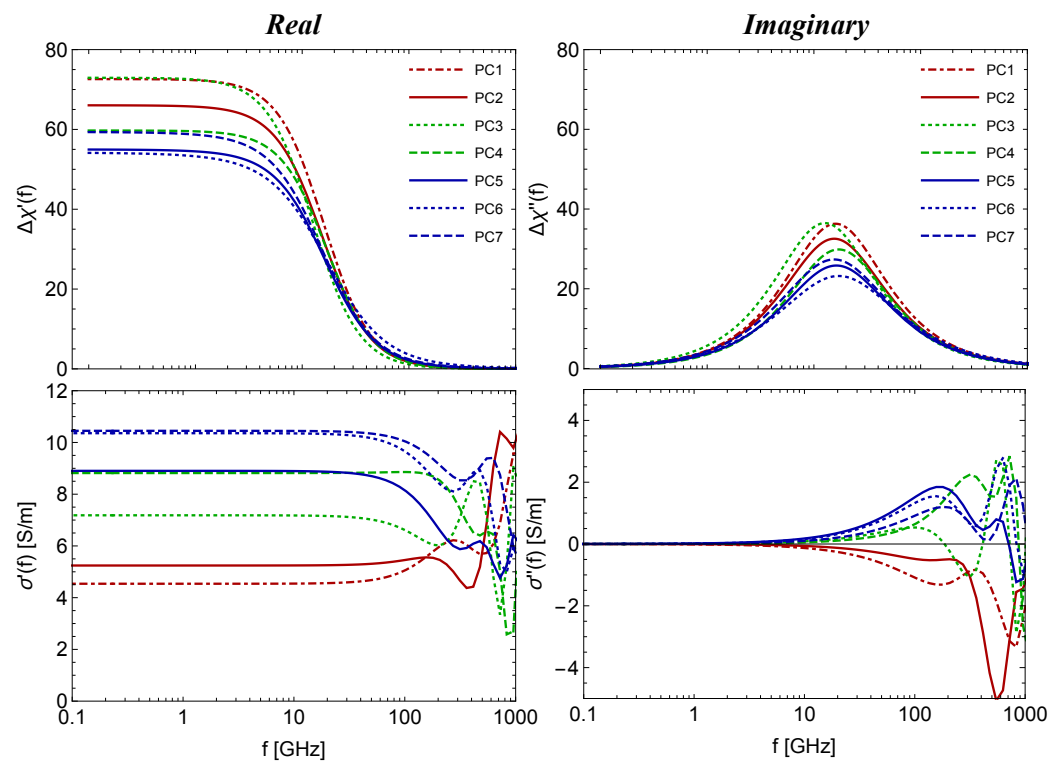

Fig. 2. Frequency-dependent susceptibility $\Delta \chi=\epsilon-1=\Delta \chi^{\prime}(f)-i \Delta \chi^{\prime \prime}(f)$ (top) and DC-conductivity $\sigma=\sigma^{\prime}(f)-i \sigma^{\prime \prime}(f)$ (bottom) of age-dependent pore solutions from correlation functions computed using MD simulations [10].

\section{$3.2 \quad$ Upscaling the electromagnetic properties}

The self-consistent scheme was used to get the effective properties of the cement paste since it captures the complex liquid-solid transition observed in cement paste at early-ages $[10,11]$. Mori-Tanaka is deployed to homogenize mortar and concrete scales, in which a clear matrix/inclusion morphology is observed. The volume fractions of sand (at the mortar scale) and of coarse aggregates (at the concrete scale) are each $40 \%$. To upscale the effective permittivity $\epsilon=\Delta \chi+1$ of the cement paste we use the Cole-Cole fits with the parameters in Tab. 1 to describe the dielectric permittivity of each pore solution $\epsilon_{P S}$. Powers cement hydration model is deployed to estimate the evolution of the capillary porosity (i.e. the volume fraction associated to the pore solution phase) in the system:

$$
f_{P S}(\xi, w / c)=\phi_{c a p}^{0}(w / c)-1.32\left(1-\phi_{c a p}^{0}(w / c)\right) \xi
$$

where the degree of hydration $\xi=0.9\left[1-e^{-t / 7}\right]$ is described by an exponential (in agreement with boundary nucleation and growth models [7]) for $t$ in days [11], and $\phi_{c a p}^{0}(w / c)=(w / c) /\left(\rho_{w} / \rho_{c}+(w / c)\right)$ is the initial porosity computed from the $w / c$ and densities of water and cement ( $\rho_{c}$ and $\rho_{w}$, respectively).

Figure 3 shows the homogenized values of the static conductivity and dielectric permittivity at the cement paste, mortar, and concrete scales. The electrical conductivity of the hydrates adopted is of $0.0246 \mathrm{~S} / \mathrm{m}$ as obtained from Monte 
Carlo Micromechanics in ref. [11]. The (non-porous- aggregates are considered perfect insulators $(\sigma(0)=0)$. The three stages associated with the pore solution composition, namely, the very early-age, early-age and late age, are retrieved in the trends observed in the static electrical properties. At the very early age, the conductivity reaches its maximum at all scales, drops drastically in the early-age stage and remains close to zero at late ages. The inverse tendency is observed with respect to the resistivity $R=1 / \sigma$ (which is the reciprocal of the conductivity). At larger scales, for a given age, the conductivity decreases. Again, the inverse is observed with the resistivity.
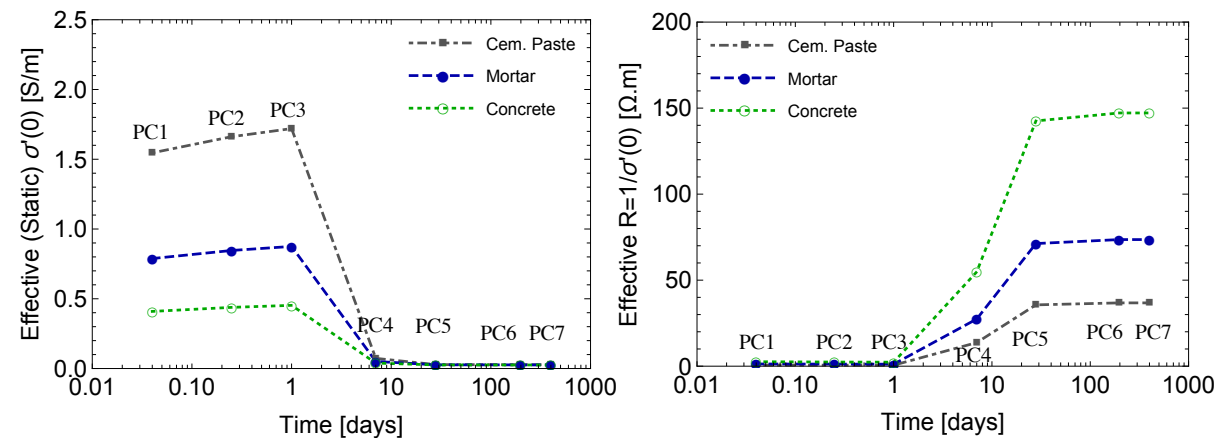

Fig. 3. Upscaling the static conductivity and resistivity at cement paste, mortar, and concrete scales. The properties of the pore solution refers to a cement paste with a $w / c$ of 0.4. We assumed a volume fraction of sand at the mortar scale of $40 \%$ and of coarse aggregate at the concrete scale of $40 \%$. The electrical conductivity of the hydrates adopted is of $0.0246 \mathrm{~S} / \mathrm{m}$ as obtained from Monte Carlo Micromechanics in ref. [11]. The aggregates are considered perfect insulators $(\sigma(0)=0)$.

Figure 4 shows the effective frequency-dependent dielectric spectra at cement paste, mortar, and concrete scales. We considered a dielectric permittivity of solids of 8 based on experimental evidence from the literature[3]. The real part of the dielectric permittivity decreases with age for frequencies below $0.05 \mathrm{GHz}$ and slightly increases with age for higher frequencies. This behavior is observed in all scales considered, being more pronounced at the cement paste scale. The imaginary part of the permittivity reaches a maximum at the very early age and for a frequency of $0.02 \mathrm{GHz}$, which shows the non-monotonous evolution of the imaginary part with the frequency. In larger scales, the differences between the results at the different frequencies are reduced, which is expected given the reduction in the relative volume fraction of cement paste present in the material at larger scales. 
T. Honorio et al.
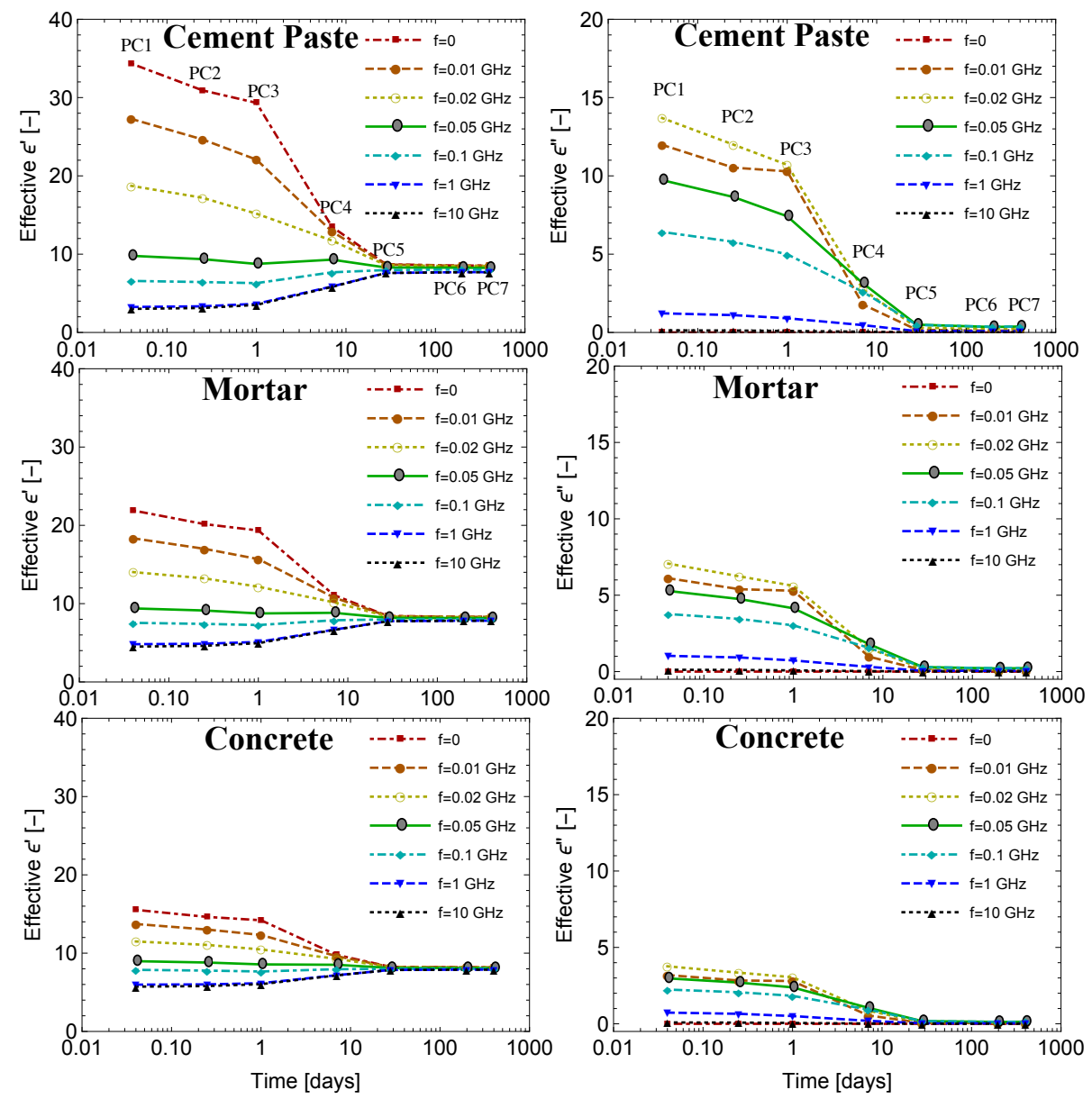

Fig. 4. Upscaling the frequency-dependent dielectric spectra $\epsilon=\epsilon^{\prime}(f)-i \epsilon^{\prime \prime}(f)$ at cement paste, mortar and concrete scales: Real part (left) and imaginary (right) parts. We assumed a volume fraction of sand at the mortar scale of $40 \%$ and of coarse aggregate at the concrete scale of $40 \%$. We considered a dielectric permittivity of solids of 8 $[3]$. 


\section{Conclusions}

The electromagnetic response of concrete is closely related to the nanoscale processes associated with water and ion interactions. The combination of molecular simulations and micromechanics provides a robust and powerful framework to link the composition and (micro-)structure to the macroscopic electromagnetic properties of cement-based materials. In this work, we showed that such a framework provided estimations of the electromagnetic properties of cementbased materials across scales that are consistent with the experimental evidence. In particular, we quantified the effects of the variability of the ionic composition of the pore solution on effective electrical conductivity and frequency-dependent dielectric response. Our results suggest that the characterization of the pore solution is a critical step in reducing the empirism of the interpretations of the resistivity and HF measurement in concrete.

\section{References}

1. Buchner, R., Hefter, G.T., May, P.M.: Dielectric Relaxation of Aqueous $\mathrm{NaCl}$ Solutions. The Journal of Physical Chemistry A 103(1), 1-9 (Jan 1999). https://doi.org/10.1021/jp982977k, http://pubs.acs.org/doi/abs/10.1021/jp982977k

2. Caillol, J.M., Levesque, D., Weis, J.J.: Theoretical calculation of ionic solution properties. The Journal of Chemical Physics 85(11), 6645-6657 (Dec 1986)

3. Guihard, V., Taillade, F., Balayssac, J.P., Steck, B., Sanahuja, J.: Permittivity measurement of cementitious materials and constituents with an open-ended coaxial probe: combination of experimental data, numerical modelling and a capacitive model. RILEM Technical Letters 4, 39-48 (Jul 2019). https://doi.org/10.21809/rilemtechlett.2019.77, https://letters.rilem.net/index.php/rilem/article/view/77

4. Honorio, T.: Monte Carlo Molecular Modeling of Temperature and Pressure Effects on the Interactions between Crystalline Calcium Silicate Hydrate Layers. Langmuir 35(11), 3907-3916 (Mar 2019). https://doi.org/10.1021/acs.langmuir.8b04156, https://doi.org/10.1021/acs.langmuir.8b04156

5. Honorio, T., Bary, B., Benboudjema, F.: Multiscale estimation of ageing viscoelastic properties of cement-based materials: A combined analytical and numerical approach to estimate the behaviour at early age. Cement and Concrete Research 85, 137-155 (Jul 2016)

6. Honorio, T., Bary, B., Benboudjema, F.: Thermal properties of cement-based materials: Multiscale estimations at early-age. Cement and Concrete Composites 87, 205-219 (Mar 2018)

7. Honorio, T., Bary, B., Benboudjema, F., Poyet, S.: Modeling hydration kinetics based on boundary nucleation and space-filling growth in a fixed confined zone. Cement and Concrete Research 83, 31-44 (May 2016)

8. Honorio, T., Bary, B., Sanahuja, J., Benboudjema, F.: Effective properties of ncoated composite spheres assemblage in an ageing linear viscoelastic framework. International Journal of Solids and Structures 124, 1-13 (Oct 2017)

9. Honorio, T., Benboudjema, F., Bore, T., Ferhat, M., Vourc'h, E.: The pore solution of cement-based materials: structure and dynamics of water and ions from molecular simulations. Physical Chemistry Chemical Physics 21, 11111-11121 (2019) 
10. Honorio, T., Bore, T., Benboudjema, F., Vourc'h, E., Ferhat, M.: Dielectric properties of the pore solution in cement-based materials. Journal of Molecular Liquids p. 112548 (Jan 2020). https://doi.org/10.1016/j.molliq.2020.112548, http://www.sciencedirect.com/science/article/pii/S0167732219360593

11. Honorio, T., Carasek, H., Cascudo, O.: Electrical properties of cementbased materials: Multiscale modeling and quantification of the variability. Construction and Building Materials 245, 118461 (Jun 2020). https://doi.org/10.1016/j.conbuildmat.2020.118461

12. Honorio, T., Lemaire, T., Tommaso, D.D., Naili, S.: Anomalous water and ion dynamics in hydroxyapatite mesopores. Computational Materials Science 156, 26 34 (Jan 2019)

13. Honorio, T., Lemaire, T., Tommaso, D.D., Naili, S.: Molecular modelling of the heat capacity and anisotropic thermal expansion of nanoporous hydroxyapatite. Materialia p. 100251 (Feb 2019)

14. Köhler, F., Findenegg, G.H.: The liquid state, vol. 1. Wiley-VCH (1972)

15. Milton, G.W.: The Theory of Composites. Cambridge University Press, Cambridge (2002)

16. Praprotnik, M., Janežič, D.: Molecular dynamics integration and molecular vibrational theory. III. The infrared spectrum of water. The Journal of Chemical Physics 122(17), 174103 (May 2005). https://doi.org/10.1063/1.1884609, http://aip.scitation.org/doi/10.1063/1.1884609

17. Rinne, K.F., Gekle, S., Netz, R.R.: Dissecting ion-specific dielectric spectra of sodium-halide solutions into solvation water and ionic contributions. The Journal of Chemical Physics 141(21), 214502 (Dec 2014)

18. Torquato, S.: Random Heterogeneous Materials: Microstructure and Macroscopic Properties. Springer Science \& Business Media (2002)

19. Vollpracht, A., Lothenbach, B., Snellings, R., Haufe, J.: The pore solution of blended cements: a review. Materials and Structures 49(8), 3341-3367 (Aug 2016)

20. Wyrzykowski, M., Sanahuja, J., Charpin, L., Königsberger, M., Hellmich, C., Pichler, B., Valentini, L., Honório, T., Smilauer, V., Hajkova, K., Ye, G., Gao, P., Dunant, C., Hilaire, A., Bishnoi, S., Azenha, M.: Numerical benchmark campaign of COST Action TU1404 - microstructural modelling. RILEM Technical Letters 2, 99-107 (Dec 2017) 